\title{
PESQUISA DE Streptococcus agalactiae NA SECREÇÃO VAGINALE ANAL DE GESTANTES DE UM MUNICÍPIO DO NOROESTE PAULISTA
}

REZENDE, Cátia. Farmacêutica-bioquímica, mestre em Biotecnologia, docente da disciplina de Microbiologia Clínica do curso de Farmácia do Centro Universitário de Votuporanga - Unifev. Campus Centro - Bloco 6 - Lab. Didático de Análises Clínicas - Rua Pernambuco, 4196. CEP: 15500-006, Votuporanga-SP.

E-mail: catia_rezende@terra.com.br.

AZEREDO, Anne; SILVEIRA, Dariane Galvão. Graduandas do curso de Farmácia do Centro Universitário de Votuporanga - Unifev.

MALTA, Roberto Carlos Grassi. Farmacêutico, mestre em Parasitologia, coordenador do curso de

Farmácia do Centro Universitário de Votuporanga - Unifev.

CASTRO, Valéria da Cruz Oliveira de. Farmacêutica, mestre em Educação em Saúde, diretora técnica em Serviços de Saúde.

MIZIARA, Renata Camacho. Biomédica, doutora em Genética, docente da disciplina de Microbiologia Clínica do curso de Farmácia do Centro Universitário da FEB.

\begin{abstract}
RESUMO
O Streptococcus agalactiae tem grande importância médica nas infecções neonatais graves associadas com morbidade e mortalidade. É a bactéria mais frequentemente isolada dos quadros de septicemia, pneumonia e meningite neonatal. Este estudo teve como objetivo determinar a prevalência de colonização anale vaginal de Streptococcus agalactiae em gestantes em diferentes idades gestacionais. A incidência de colonização por $S$. agalactiae foi avaliada em 129 gestantes de qualquer idade gestacional. Foram coletadas duas amostras de secreção para cultura: um swab perianal e um swab vaginal. Cada um dos dois swabs foi inoculado em tubo de ensaio contendo caldo de Todd-Hewitt e subcultivados em ágar sangue. Após 24 a 48 horas, as colônias sugestivas de Streptococcus agalactiae foram submetidas à análise morfotintorial e a provas bioquímicas para identificação. Das 129 gestantes analisadas, 3 (2,33\%) tiverem cultura positiva para Streptococcus agalactiae e $126(97,67 \%)$ apresentaram resultado negativo. Os resultados apresentados neste trabalho estão abaixo dos dados de outros estudos, entretanto, a metodologia utilizada foi compatível com a maioria dos autores. Ainda assim, essa taxa é considerada um valor relevante, tendo em vista a importância do Streptococcus agalactiae em infecções em gestantes e neonatos, ressaltando a necessidade de estimular a cultura de secreção vaginal e anal para pesquisa de Streptococcus agalactiae nos exames de pré-natal.
\end{abstract}

Palavras-chave: Streptococcus agalactiae; Gestante; Colonização.

\begin{abstract}
Streptococcus agalactiae has great medical importance in infections associated with severe neonatal morbidity and mortality. It is the most frequent bacterium isolated from the tables of septicemia, pneumonia and neonatal meningitis. This study aimed to determine the prevalence of anal and vaginal colonization of $S$. agalactiae in pregnant women at different gestational ages. We evaluated the incidence of colonization by $S$. agalactiae in 129 pregnant women of any age. Two samples of secretion were collected for culture: a perianal swab and a vaginal swab. Each of the two swabs were inoculated in test tubes containing Todd-Hewitt broth and subcultured on blood agar. After a period of 24 to 48 hours, the colonies suggestive of $S$. agalactiae were submitted to morfotintorial analysis and to biochemical tests for identification. Among 129 women studied, 3 (2.33\%) have tested positive for Streptococcus agalactiae and $126(97.67 \%)$ were negative. The results presented in this
\end{abstract}


work are inferior to the data from other studies, however, the methodology used was compatible with most authors. Even so this rate is considered a relevant value taking into account the importance of Streptococcus agalactiae infections in pregnant women and newborns, emphasizing the importance of encouraging the culture of vaginal and anal secretion for the detection of Streptococcus agalactiae in surveys of prenatal care.

Keywords: Streptococcus agalactiae; Pregnant woman; Colonization.

\section{INTRODUÇÃo}

O Streptococcus agalactiae faz parte da microbiota de membranas mucosas de seres humanos e animais, colonizando principalmente o trato intestinal e geniturinário. Também conhecido como estreptococo do grupo B-EGB, a grande importância médica deste microrganismo está nas infecções neonatais graves, septicemia, pneumonia e meningite neonatal, associadas com morbidade e mortalidade (SCHRAG et al., 2002). Além disso, pode causar infecção no organismo materno, comprometendo a evolução da gestação, provocando aborto, infecção urinária, prematuridade, corioamnionite e endometrite puerperal, que podem ser prevenidos pela identificação e tratamento das gestantes colonizadas (OVALLE et al., 2002; GIBBS; SCHRAG; SCHUCHAT, 2004).

$\mathrm{O}$ contato com o agente infectante geralmente ocorre na passagem pelo canal vaginal durante o parto (BÁRBAROS et al., 2005). Cerca de 50 a $75 \%$ dos recém-nascidos - RN expostos ao EGB intravaginal tornam-se colonizados, e 1 a $2 \%$ de todos $\mathrm{RN}$ de mães portadoras desenvolverão doença invasiva de início precoce (MERCOLA, 2001).

$O$ índice de isolamento do microrganismo depende de vários fatores relacionados com a coleta e a metodologia laboratorial empregadas (QUINLAN et al., 2000). A cultura de triagem deve ser realizada entre a $35 .^{\mathrm{a}}$ e a $37 .^{\mathrm{a}}$ semanas de gestação, considerado o período no qual se demonstrou melhor sensibilidade e especificidade para a detecção do EGB (SCHRAG et al., 2002; MONEY; DOBSON, 2004). A coleta tem maior eficácia quando realizada com dois swabs - vaginal (terço inferior do trato genital) e anal (dentro do esfíncter anal). Não são aceitáveis culturas cervicais nem amostras colhidas com espéculo. A utilização de meio seletivo, contendo agentes antimicrobianos para inibir o crescimento de outros microrganismos, aumenta a possibilidade de crescimento do EGB em aproximadamente 50\% (SCHRAG et al., 2002).

Nos Estados Unidos, no início da década de 1970, esse agente foi identificado como o principal responsável pela meningite e septicemia em recémnascidos (PLATT; O'BRIEN, 2003). Na década seguinte, demonstrou-se que os índices de infecção neonatal pelo agente foram bastante reduzidos, depois de instituída a antibioticoterapia durante o parto para as mulheres de risco (LARSEN; SEVER, 2008). Diante disso, medidas profiláticas têm sido incentivadas em nível internacional, com o intuito de diminuir a incidência das infecções e suas possíveis consequências ao neonato (POGERE et al., 2005).

Os antibióticos habitualmente utilizados na quimioprofilaxia são penicilina ou ampicilina e, em casos de alergia, eritromicina ou clindamicina. No entanto, vários estudos, em diferentes países, têm mostrado o aparecimento de cepas de EGB resistentes a estes últimos antimicrobianos, o que os torna inadequados como opção de escolha tanto para a quimioprofilaxia como para o tratamento de infecções (DEAZEVEDO et al., 2001; D'OLIVEIRA et al., 2003).

No Brasil, o Streptococcus agalactiae não tem sido ainda devidamente valorizado na etiologia dos processos infecciosos, apesar da gravidade da infecção e de a mesma ser passível de benefícios profiláticos (QUINLAN et al., 2000). Considerando que há pouca atenção dos órgãos responsáveis pela prevenção, este trabalho teve como objetivo determinar a prevalência de colonização anal e vaginal de Streptococcus agalactiae em gestantes em diferentes idades gestacionais, visando contribuir com informações sobre tal assunto, que ainda são raras em nosso meio. 


\section{Metodologia}

Um estudo transversal foi realizado entre junho e novembro de 2009 em 129 gestantes de qualquer idade gestacional, primíparas ou multíparas, sem sinais clínicos de infecção no momento da coleta. A incidência de colonização por Streptococcus agalactiae foi avaliada. O projeto foi aprovado pelo Comitê de Ética em Pesquisa do Centro Universitário de Votuporanga - UNIFEV (Protocolo n. ${ }^{\circ}$ 010/2009).

Foram incluídas neste estudo pacientes de um laboratório de análises clínicas que atende ao Sistema Único de Saúde -SUS e aos convênios, e de Unidades Básicas de Saúde de um município do noroeste paulista. Os critérios de exclusão foram: realização de exame ginecológico, no mesmo dia ou anteriormente à coleta, ou utilização de antibióticos há pelo menos duas semanas, devido à possibilidade de um resultado falso negativo. As coletas e o processamento dos materiais estéreis e descartáveis foram realizados de acordo com as recomendações do Centers for Disease Control and Prevention - CDC (CDC, 2004).

Todas as gestantes concordaram em participar do estudo e assinaram o Termo de Consentimento Livre e Esclarecido - TCLE. Um questionário sobre o número de gestações e ocorrência de abortos foi respondido por todas as pacientes. As gestantes foram submetidas à coleta de duas amostras de secreção para cultura: um swab perianal e um swab vaginal (no terço distal da vagina).

Cada um dos dois swabs foi inoculado em tubo de ensaio individual, devidamente identificado, contendo meio de cultura seletivo, caldo de Todd-Hewitt suplementado com $10 \mu \mathrm{g} / \mathrm{mL}$ de colistina e $15 \mu \mathrm{g} / \mathrm{mL}$ de ácido nalidíxico, e transportados adequadamente para o Laboratório de Análises Clínicas do Centro Universitário de Votuporanga-Unifev. Após incubação de 18 a 24 horas a $35^{\circ} \mathrm{C}$, foram subcultivados em ágar sangue de carneiro desfribrinado. Após 24 a 48 horas de incubação em atmosfera de microaerofilia, as placas foram inspecionadas e as colônias sugestivas de EGB, acinzentadas, circundadas ou não por halo discreto de hemólise total (ß-hemólise), foram submetidas à análise morfotintorial. As colônias que apresentavam morfologia compatível com cocos Gram-positivos foram semeadas em ágar BHI e submetidas às provas bioquímicas de catalase e CAMP. Foi avaliada também a relação da colonização por EGB com o número de gestações e abortos de cada gestante.

\section{Resultados}

Das 129 gestantes analisadas, 3 (2,33\%) tiveram cultura positiva para Streptococcus agalactiae e 126 $(97,67 \%)$ apresentaram resultado negativo. Todas as gestantes tiveram cultura positiva nos dois sítios - anal e vaginal (Tabela 1$)$.

Tabela 1 - Colonização por Streptococcus agalactiae nos sítios anale vaginal.

\begin{tabular}{ccc}
\hline Sítio Anatômico & Positivo para EGB/Total & $\%$ \\
\hline Vaginal + Anal & $3 / 129$ & 2,33 \\
\hline
\end{tabular}

$\mathrm{EGB}=$ Streptococcus agalactiae

Fonte: Dados de pesquisa.

A faixa etária variou entre 19 e 39 anos, não havendo diferença significativa entre a ocorrência de cultura positiva e a idade das pacientes (ver Tabela 2). 
Pesquisa de Streptococcus agalactiae...

Tabela 2 - Colonização por Streptococcus agalactiae de acordo com a faixa etária.

\begin{tabular}{ccc}
\hline Faixa Etária & Positivo para EGB/Total & $\%$ \\
\hline $19-24$ & $2 / 57$ & 3,51 \\
$25-30$ & $1 / 49$ & 2,04 \\
Acima de 31 & $0 / 23$ & 0 \\
\hline
\end{tabular}

EGB $=$ Streptococcus agalactiae

Fonte: Dados de pesquisa.

Das gestantes colonizadas por EGB, 2 tiveram apenas uma gestação e 1 teve duas gestações (Tabela 3).

Tabela 3 - Colonização por Streptococcus agalactiae de acordo com o número de gestações.

\begin{tabular}{ccc}
\hline Número de gestações & Positivo para EGB/Total & $\%$ \\
\hline 1 & $2 / 52$ & 3,85 \\
2 & $1 / 35$ & 2,86 \\
4 & $0 / 27$ & 0 \\
4 ou mais & $0 / 15$ & 0 \\
\hline
\end{tabular}

$\mathrm{EGB}=$ Streptococcus agalactiae

Fonte: Dados de pesquisa.

As três gestantes com resultado positivo não os dados não são considerados significativos relataram ocorrência de aborto (Tabela 4). Ainda assim, estatisticamente.

Tabela 4 - Colonização por Streptococcus agalactiae de acordo com a ocorrência de aborto.

\begin{tabular}{ccc}
\hline Aborto & Positivo para EGB/Total & $\%$ \\
\hline Sim & $0 / 42$ & 0 \\
Não & $3 / 87$ & 3,45 \\
\hline
\end{tabular}

$\mathrm{EGB}=$ Streptococcus agalactiae

Fonte: Dados de pesquisa. 


\section{Discussão}

Os resultados apresentados neste trabalho apontaram uma prevalência de colonização por Streptococcus agalactiae (2,33\%) em gestantes, abaixo dos valores obtidos por outros autores. A taxa de colonização por estreptococo do grupo B na vagina e no reto varia de 5 a 40\% (GRIMWOOD et al., 2002). Sabe-se também que a prevalência do EGB é relacionada a outras variáveis como: idade, paridade, localização geográfica, metodologia e nível socioeconômico. Por isso, as taxas de colonização por EGB podem ter resultados diferentes em diversas localidades como: Turquia (8\%), Estados Unidos (18,6 a 21,1\%), Chile (19,9\%), Índia/Paquistão (12\%), Ásia/Pacífico (19\%), África (19\%), Norte da África (22\%) (BELMAR et al., 2002; BARBAROS et al., 2005; POGERE et al., 2005).

No Brasil, a incidência de Streptococcus agalactiae, revelada por pesquisas já realizadas, mostraram uma variação de 15 a 25\%. Emestudos em Londrina, Paraná, foi encontrada uma taxa de $15 \%$ de colonização entre 100 mulheres avaliadas (MOCELIN et al., 1995). Estudos mais recentes realizados em Santa Catarina e no Paraná encontraram prevalências de 21,6\% em 273 gestantes e 14,9\% em 309 gestantes, respectivamente (BERALDO et al., 2004; POGERE et al., 2005). Apesar da diferença estatística, os trabalhos de Beraldo e colaboradores (2004) e Pogere e colaboradores (2005) utilizaram a mesma metodologia: coleta vaginal e anorretal, utilizando dois swabs que foram inoculados separadamente em meio seletivo e incubados por 18 a 24 horas.

Em São Luís, Maranhão, entre 2005 e 2006, realizou-se um estudo sobre a prevalência de colonização materna pelo EGB com 201 mulheres e foi encontrada uma taxa de 20,4\%. A metodologia utilizada por Costa e colaboradores (2008) neste estudo foi semelhante aos métodos anteriores, porém com incubação de 24 horas. Outro trabalho realizado na Universidade Federal Fluminense, no Rio de Janeiro, encontrou uma prevalência de 19,2\% em 167 grávidas. Borger e colaboradores (2005) utilizaram apenas um $s w a b$ nas culturas vaginal e anal, com incubação no meio seletivo por seis horas. No entanto, obteve taxa de positividade semelhante a outros estudos.

A prevalência de colonização mostrada neste estudo $(2,33 \%)$ está abaixo dos demais trabalhos pesquisados. Entretanto, a metodologia utilizada é compatível com a maioria dos autores: coleta nos sítios vaginal e anal, com dois swabs e inoculação separada em meio seletivo por 18 a 24 horas. Mas, ainda assim, a taxa é considerada um valor relevante, tendo em vista a importância do Streptococcus agalactiae em infecções em gestantes e neonatos. As taxas de colonização por EGB em comparação com a faixa etária, ocorrência de abortos e número de gestações não tiveram diferenças significativas, portanto, estes fatores não devem ser considerados no critério para escolha da quimioprofilaxia.

No Brasil os dados são escassos, e a principal falha encontrada em muitas regiões é a não utilização de meios seletivos e coleta apenas do sítio vaginal, excluindo-se o anal (POGERE et al., 2005). Isso pode ser considerado preocupante, já que é provável que taxas elevadas de infecção neonatal estejam ocorrendo sem serem identificadas.

Em estudo feito em São Paulo, as taxas detectadas foram baixas, de 1,9\% (BELMAR et al., 2002); e em um trabalho realizado em Salvador, o índice foi de 6,9\% (PELLEGRINI, 1999), ambos semelhantes ao detectado neste trabalho. Sabe-se que a coleta realizada nas regiões vaginal e retal aumenta a sensibilidade do exame; portanto, esses resultados podem ter sido em decorrência da coleta apenas vaginal e da inoculação em meio de cultura não seletivo, já que os dois trabalhos utilizaram a mesma metodologia (SCHRAG et al., 2002).

Vários estudos têm demonstrado alguns casos positivos apenas no sítio anal; assim, esses casos deixariam de ser diagnosticados se a coleta não tivesse sido realizada nos dois sítios (BERALDO et al., 2004; POGERE et al., 2005; COSTA et al., 2008). Quando é utilizada a associação dos dois sítios de coleta, há um aumento da detecção do EGB de 5 a $25 \%$ (CDC, 2004). Diante disso, parece que o meio seletivo e os sítios de coleta são fatores que levam a 
um diagnóstico seguro da infecção pelo EGB e, quando utilizados, as taxas de prevalência de colonização são semelhantes em todos os trabalhos encontrados (POGERE et al., 2005).

Um estudo realizado em Ribeirão Preto, São Paulo, em 1997, demonstrou que o EGB foi a bactéria mais prevalente nos casos de septicemia neonatal, estando presente em $37,8 \%$ dos casos de infecção confirmada (NOBRE, 1997). Assim como em Porto Alegre, Rio Grande do Sul, em 2001, onde foi demonstrado que o Streptococcus agalactiae era um dos patógenos mais importantes na etiologia de septicemia de início precoce em neonatos, comincidência de $1 / 1000$ nascidos vivos (MIURA; MARTIN, 2001).

A epidemiologia da infecção por Streptococcus agalactiae em mulheres grávidas e neonatos tem sido muito estudada nos Estados Unidos e Europa, mas o mesmo não ocorre na América Latina (ROWEN; BAKER, 1998). Ainda assim, as taxas de prevalência descritas nos estudos brasileiros, mesmo que subestimadas, levam a uma reflexão sobre a importância de uma estratégia de profilaxia das mães e recém-nascidos. Os custos com internações e terapias para os neonatos acometidos por esta patologia podem ter maior impacto financeiro do que estabelecer um protocolo de profilaxia (AKKER-VAN MARLE et al., 2005).

\section{Conclusão}

Portanto, baseado na frequência de colonização verificada neste estudo (2,33\%) e em vários outros, ressalta-se a importância de estimular os profissionais da saúde, a incluir a cultura de secreção vaginal e anal para pesquisa de EGB nos exames pré-natais. A identificação de mulheres colonizadas permitirá a aplicação endovenosa profilática de antibióticos antes ou durante o parto. Dessa forma, seriam reduzidas as infecções neonatais, contribuindo para uma melhor qualidade de vida da população.

\section{REFERÊNCIAS}

AKKER-VAN MARLE, M.E.; RIJNDERS, M.E.;
DOMMELEN, P.; FEKKES, M.; WOUWE, J.P.; AMELINK-VERBURG, M.P.; et al. Costeffectiviness of different treatment strategies with intrapartum antibiotic prophylaxis to prevent earlyonset group B streptococcal disease. BJOG, v.112, n.6, p.820-6, 2005.

BARBAROS, I.; MURAT, C.; MEHMET, V.; ISMET, T.A.; CAN, K.; SUKUFE, D.; et al. The colonization incidence of group B streptococcus in pregnant women and their newborns in Istanbul.

Pediatr. Int., v.47, n. 1, p.6-64, 2005.

BELMAR, J.C.; ABARZÚA, C.F.; BEKER, V.J.; GUSMÁN, A.M.; GARCÍA, C.P.; OYARZÚN EBENSPERGER, E. Estudio de sensibilidad antimicrobiana de 183 cepas de Streptococcus agalactiae aisladas en región vagino-perineal de embarazadas en el tercer trimeste. Ver. Chil. Obstet. Ginecol., v.67, n.2, p.106-9, 2002.

BERALDO, C.; BRITO, A.S.J.; SARIDAKIS, H.O.; MATSUO, T. Prevalência da colonização vaginal e anorretal por estreptococo do grupo B em gestantes do terceiro trimestre. Ver. Bras. Ginecol. Obstet., v.26, n.7, p.543-549, 2004.

BORGER, I.L.; D'OLIVEIRA, R.E.C.; CASTRO, A.C.D.; MONDINO, S.S.B. Streptococcus agalactiae em gestantes: prevalência de colonização e avaliação da suscetibilidade aos antimicrobianos.

Rev. Bras. Ginecol. Obstet., v.27, n.10, p.575579, 2005.

\section{CENTERS FOR DISEASE CONTROLAND} PREVENTION (CDC). Laboratory practices for prenatal group B streptococcal screening-seven states, 2003. MMRW Morb. Mortal Wkly Rep, v.53, n.23, p.506-9, 2004.

COSTA, A.L.R.; LAMY FILHO, F.; CHEIN, M.B.C.; BRITO, L.M.O.; LAMY, Z.C.;

ANDRADE, K.L. Prevalência de colonização por 
estreptococos do grupo B em gestantes atendidas em maternidade pública da região Nordeste do Brasil. Rev Bras Ginecol. Obstet., v.30, n.6, p.274-280, 2008.

D'OLIVEIRA, R.E.; BARROS, R.R.; MENDONÇA, C.R.; TEIXEIRA, L.M.; CASTRO, A.C. Susceptibility to antimicrobials and mechanisms of erythromycin resistance in clinical isolates of Streptococcus agalactiae from Rio de Janeiro, Brazil. J Med. Microbiol., v.52, n.11, p.1029-30, 2003.

DE AZAVEDO, J.C.; MCGAVIN, M.; DUNCAN, C.; LOW, D.E.; MCGEER, A. Prevalence and mechanisms of macrolide resistance in invasive and noninvasive group B streptococcus isolates from Ontario, Canada. Antimicrob. Agents.

Chemother. v.45, n.12, p.3504-8, 2001.

GIBBS, R.S.; SCHRAG, S.; SCHUCHAT, A. Perinatal infections due to group B streptococci.

Obstet. Gynecol., v.104, n.5, p.1062-76, 2004.

GRIMWOOD, K.; STONE, P.R.; GOSLING, I.A.; et al. Late antenatal carriage of group B Streptococcus by New Zealand women. Aust. N. Z. J. Obstet Gynaecol, v.42, p.182-6, 2002.

LARSEN, J.W.; SEVER, J.L. Group B streptococcus and pregnancy: a review. Am. J ...Obstet. Gynecol., v.198, n.4, p.440-8, 2008.

MERCOLA, J. Group B streptococcus. Midwifery Today E-News, v.3, n.37, p.12, 2001.

MIURA, E.; MARTIN, M.C. Group B streptococcal neonatal infections in Rio Grande do Sul, Brazil. Rev. Inst Med. Trop. Sao Paulo, v.43, n.5, p.243-6, 2001.

MOCELIN, C.O.; CARVALHO, D.A.F.; BRITES, C.; CHRISTOFOLLI, D.; MOCELIN, A.O.;
FRACALANZZA, S.E.L.; et al. Isolamento de Streptococcus agalactiae de gestantes na região Londrina-PR. Rev. Bras. Ginecol. Obstet., v.17, n.9, p.915-8, 1995.

MONEY, D.M.; DOBSON, S. The prevention of early-onset neonatal group B streptococcal disease. J. Obstet. Gynecol. Can., v.26, n.9, p.826-40, 2004.

NOBRE, RA. Frequência de infecção por streptococcus b-hemolítico do grupo $B$ e outras bactérias em recém-nascidos com desconforto respiratório. 1997. Ribeirão Preto: Universidade de São Paulo; 1997.

OVALLE SALAS, A.; GÓMEZ, M.R.; MARTINEZ TAGLE, M.A.; ASPILLAGA MANTEROLA, C.; DOLZ, C.S. Infección vaginal $\mathrm{y}$ tratamiento del streptococcus grupo $\mathrm{B}$ en embarazadas con factores universales de riesgo de infección: resultados neonatales y factores de riesgo de infección neonatal. Rev. Chil. Obstet. Ginecol., v.67, n.6, p.465-75, 2002.

PELLEGRINI, R. Frequência de colonização por Streptococccus agalactiae em gestantes da cidade de Salvador, Bahia. Rev. Soc Bras. Med. Trop., v.32, n.4, p.451-2, 1999.

PLATT, J.S.; O'BRIEN, W.F. Group B streptococcus: prevention of early-onset neonatal sepsis. Obstet. Gynecol. Surv., v.58, n.3, p.191-6, 2003.

POGERE, A.; ZOCCOLI, C.M.; TOBOUTI, N.R.; FREITAS, P.F.; D'ACAMPORA, A.J.; ZUNINO, J.N. Prevalência da colonização pelo estreptococo do grupo B em gestantes atendidas em ambulatório de pré-natal. Rev. Bras. Ginecol. Obstet., v.27, n.4, p.174-180, 2005.

QUINLAN, J.D.; HILL, D.A.; MAXWELL, B.D.; 
BOONE, S.; HOOVER, F.; LENSE, J.J. The necessity of both anorectal and vaginal cultures for group B streptococcus screening during pregnancy.

J. Fam. Pract. v.49, n.5, p.447-8, 2000.

ROWEN, J.L.; BAKER, C.J. Group B streptococcal infections. In: Feigin RD, editor. Textbook of
Pediatric Infections Diseases. 4th ed. Philadelphia: W B Saunders; 1998.v.1 p. 1089-106.

SCHRAG, S.; GORWITZ, R.; FULTZ-BULTZ, K.; SCHUCHAT, A. Prevention of perinatal group B streptococcal disease: revised guidelines from CDC. MMWR Recomm Rep, v.51, n.11, p.1-22, 2002.

RECEBIDO EM 26/10/2010

Асегто ем 4/12/2010 